\title{
Support and density of the limit m-ary search trees distribution
}

\author{
Brigitte Chauvin 1]! Quansheng Liu非 and Nicolas Pouyanne 1 非 \\ ${ }^{1}$ Université de Versailles-St-Quentin, Laboratoire de Mathématiques de Versailles, CNRS, UMR 8100 \\ ${ }^{2}$ Université de Bretagne Sud, Laboratoire de Mathématiques de Bretagne Atlantique, CNRS, UMR 6205
}

The space requirements of an $m$-ary search tree satisfy a well-known phase transition: when $m \leq 26$, the second order asymptotics is Gaussian. When $m \geq 27$, it is not Gaussian any longer and a limit $W$ of a complex-valued martingale arises. We show that the distribution of $W$ has a square integrable density on the complex plane, that its support is the whole complex plane, and that it has finite exponential moments. The proofs are based on the study of the distributional equation $W \stackrel{\mathcal{L}}{=} \sum_{k=1}^{m} V_{k}^{\lambda} W_{k}$, where $V_{1}, \ldots, V_{m}$ are the spacings of $(m-1)$ independent random variables uniformly distributed on $[0,1], W_{1}, \ldots, W_{m}$ are independent copies of $\mathrm{W}$ which are also independent of $\left(V_{1}, \ldots, V_{m}\right)$ and $\lambda$ is a complex number.

Keywords: $m$-ary search trees. Characteristic function. Smoothing transformation. Absolute continuity. Support. Exponential moments. Mandelbrot cascades.

\section{Introduction}

Search trees are fundamental data structures in computer science used in searching and sorting. For integers $m \geq 2, m$-ary search trees generalize the binary search tree. The quantity $m$ is called the branching factor.

A random $m$-ary search tree is an $m$-ary tree in which each node has the capacity to contain $(m-1)$ elements called the data or the keys. The keys can be considered as i.i.d. random variables $x_{i}, i \geq 1$, with any absolutely continuous distribution on the interval $[0,1]$.

The tree $T_{n}, n \geq 0$, is recursively defined as follows: $T_{0}$ has an empty node-root; $T_{1}$ has a noderoot which contains $x_{1} ; T_{2}$ has a node-root which contains $x_{1}$ and $x_{2} ; \ldots T_{m-1}$ still has one node-root, containing $x_{1}, \ldots x_{m-1}$. As soon as the $(m-1)$-th key is inserted in the root, $m$ empty subtrees of the root are created, corresponding from left to right to the $m$ ordered intervals $\left.I_{1}=\right] 0, x_{(1)}\left[, \ldots, I_{m}=\right] x_{(m-1)}, 1[$, where $0<x_{(1)}<\cdots<x_{(m-1)}<1$ are the ordered first $(m-1)$ keys. Each following key $x_{m}, x_{m+1}, \ldots$ is recursively inserted in the subtree corresponding to the unique interval $I_{j}$ to which it belongs. As soon as a node is saturated, $m$ empty subtrees of this node are created.

\footnotetext{
†Email: brigitte. chauvineuvsq. fr.

¥Email: quansheng. liu@univ-ubs. fr.

$\S$ Email: nicolas.pouyanne@uvsq.fr.
} 
For each $i=\{1, \ldots, m-1\}$ and $n \geq 1, X_{n}^{(i)}$ is the number of nodes in $T_{n}$ which contain $(i-1)$ keys (and $i$ gaps or free places) after insertion of the $n$-th key; such nodes are named nodes of type $i$. We only take into consideration the external nodes and not the internal nodes which are the saturated nodes. The vector $X_{n}$ is called the composition vector of the $m$-ary search tree. It provides a model for the space requirement of the algorithm. By spreading the input data in $m$ directions instead of only 2 , as is the case for a binary search tree, one seeks to have shorter path lengths and thus quicker searches. One can refer to Mahmoud's book [Mah92] for further details on search trees.

The following figure is an example of 4 -ary search tree obtained by insertion of the successive numbers $0.3,0.1,0.4,0.15,0.9,0.2,0.6,0.5,0.35,0.8,0.97,0.93,0.23,0.84,0.62,0.64,0.33,0.83$. The corresponding composition vector is $X_{18}={ }^{t}(9,2,2)$.

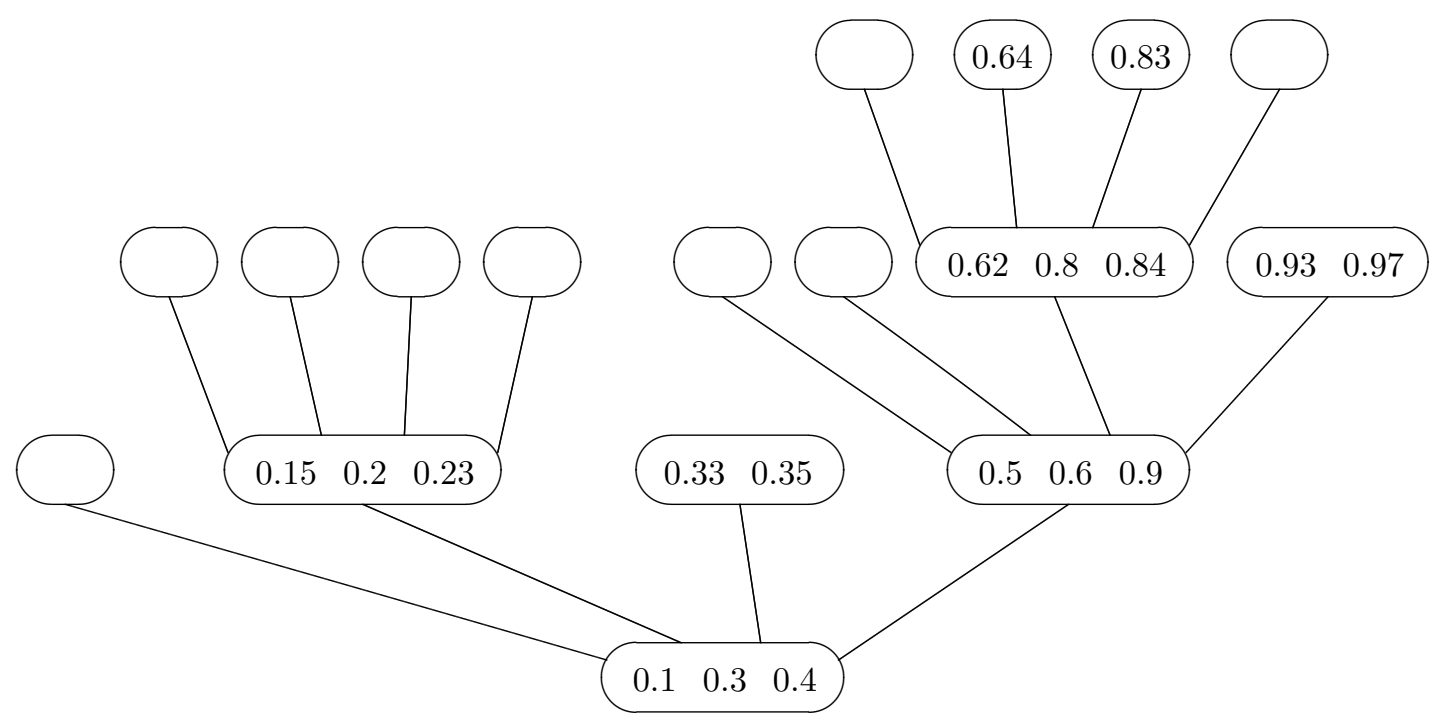

A vast literature is devoted to the asymptotic behavior of this composition vector. A famous phase transition appears. When $m \leq 26$, the random vector admits a central limit theorem with convergence in distribution to a Gaussian vector: see also Mahmoud's book [Mah92] or Janson [Jan04] for a vectorial treatment.

When $m \geq 27$, an almost sure asymptotics for the composition vector has been obtained in [CP04]:

$$
X_{n}=n v_{1}+\Re\left(n^{\lambda_{2}} W v_{2}\right)+o\left(n^{\sigma_{2}}\right) \quad \text { a.s. }
$$

(throughout the paper we write $\Re(z)$ or $\Re z$ for the real part of $z$, and $\Im(z)$ or $\Im z$ for the imaginal part of $z$ ), where $\lambda_{2}=\sigma_{2}+i \tau_{2}$ is the root of the polynomial

$$
\prod_{k=1}^{m-1}(z+k)-m !
$$


having the second largest real part $\sigma_{2}$ and a positive imaginary part $\tau_{2}, v_{1}$ and $v_{2}$ are two deterministic vectors, and $W$ is the limit of a complex-valued martingale that admits moments of all positive orders.

Heated conjectures about the second order complex-valued limit distribution $W$ remain open (see [CP04], [Pou05], Chern and Hwang [CH01], Janson [Jan04]). Namely, what is the true order of magnitude of $o\left(n^{\sigma_{2}}\right)$ in $(1)$ ? Can the $W$ distribution be expressed by means of usual distributions? How heavy are the tails of $W$ ? Can one identify the distributions of $|W|$ and $\operatorname{Arg}(W)$ ?

A significant step is achieved by Fill and Kapur in [FK04], who establish that $W$ satisfies the following distributional equation called the smoothing equation:

$$
W \stackrel{\mathcal{L}}{=} \sum_{k=1}^{m} V_{k}^{\lambda_{2}} W_{k},
$$

where $V_{1}, \ldots, V_{m}$ are the spacings of $(m-1)$ independent random variables uniformly distributed on $[0,1], W_{1}, \ldots, W_{m}$ are independent copies of $W$ which are also independent of $\left(V_{1}, \ldots, V_{m}\right)$. The precise definition of $V_{j}$ will be given hereunder. By a contraction method, Fill and Kapur prove that $W$ is the unique solution of Eq. (3) in the space $\mathcal{M}_{2}(C)$ of square integrable probability measures having $C=$ $\mathbb{E}(W)$ as expectation. The present paper is based on this characterization of $W$.

It has been recently proved [CLP11] that the continuous-time embedding of the process $\left(X_{n}\right)_{n}$ has an analogous asymptotic behavior, with a second-order term which is a solution of some distributional equation (not the same one). Inspired by this study of the continuous-time case we prove the following theorem.

Theorem 1 Let $W$ be the second order limit distribution of an $m$-ary search tree for $m \geq 27$, defined by (1).

(i) The support of $W$ is the whole complex plane.

(ii) The law of $W$ admits a continuous square integrable density on $\mathbb{C}$.

(iii) $\mathbb{E} e^{\delta|W|}<\infty$ for some $\delta>0$. The exponential moment generating series of $W$ (thus) has a positive radius of convergence.

Thanks to Fill and Kapur results [FK04], these results are immediate corollaries of Theorems 3 and 6 proved in the next two sections.

In the sequel, let $V_{1}, \ldots, V_{m}$ be the spacings of $(m-1)$ independent random variables uniformly distributed on $[0,1]$. In other words, let $U_{1}, \ldots, U_{m-1}$ be independent random variables uniformly distributed on $[0,1]$ and let $U_{(1)} \leq \cdots \leq U_{(m-1)}$ be their order statistics. Denote also $U_{(0)}:=0, U_{(m)}:=1$. For any $k \in\{1, \ldots, m\}$, the random variable $V_{k}$ is defined by

$$
V_{k}:=U_{(k)}-U_{(k-1)}
$$

The variables $V_{k}$ are $\operatorname{Beta}(1, m-1)$-distributed and satisfy $\sum_{k=1}^{m} V_{k}=1$ almost surely.

Remark 2 Details about roots of (2) can be found in Hennequin [Hen91] and Mahmoud [Mah92]. Note that for $m=2$, the polynomial (2) has the unique root $\lambda=1$. For $m \geq 3$, it is known that if $\lambda_{2}$ is a root 
of the polynomial (2) having the second largest real part, then $\lambda_{2}$ is nonreal, $\Re \lambda_{2}<1$ for any $m \geq 3$, $\Re \lambda_{2}>0$ if and only if $m \geq 14$ (relate this to Hwang [Hwa03]), and

$$
\Re \lambda_{2}>\frac{1}{2} \Longleftrightarrow m \geq 27
$$

\section{Support}

The limit distribution $W$ satisfies $(3$ ). From now on, we consider the solutions of the distributional equation

$$
Z \stackrel{\mathcal{L}}{=} \sum_{k=1}^{m} V_{k}^{\lambda} Z_{k}
$$

where $V_{1}, \ldots, V_{m}$ are the spacings of $(m-1)$ independent random variables uniformly distributed on $[0,1], Z_{1}, \ldots, Z_{m}$ are independent copies of $Z$ which are also independent of $\left(V_{1}, \ldots, V_{m}\right)$ and $\lambda$ is a nonreal complex number.

We assume that

$$
\lambda \text { is a nonreal root of (2) having a positive real part } \sigma \text {. }
$$

Indeed, $V_{1}, \ldots, V_{m}$ are $\operatorname{Beta}(1, m-1)$-distributed and $\Re(\lambda)>0$ guarantees that $\mathbb{E}\left|V_{1}^{\lambda}\right|<\infty$. Moreover, we are interested in solutions of (4) having a nonzero expectation and the existence of such solutions implies that $\lambda$ is a root of (2). Note that when $m \leq 13$, no $\lambda$ satisfies (5).

The following theorem implies Theorem 1 (i) because $W$ is integrable with $\mathbb{E} W=\frac{1}{\Gamma\left(1+\lambda_{2}\right)} \neq 0$ (see [Pou05] for instance).

Theorem 3 Let $\lambda$ be a nonreal complex number having a positive real part. If $Z$ is a solution of (4) having a nonzero expectation, then the support of $Z$ is the whole complex plane.

The proofs of Theorem 3 and Theorem 6 make use of the complex-valued random variable

$$
A:=\sum_{k=1}^{m} V_{k}^{\lambda}
$$

Notice that the existence of an integrable solution $Z$ of 4 such that $\mathbb{E}(Z) \neq 0$ implies that

$$
\mathbb{E}(A)=1,
$$

which just means that $\lambda$ is a root of the polynomial 2).

Proof of TheOREM 3 . For a complex valued random variable $X$, we denote its support by

$$
\operatorname{Supp}(X)=\{x \in \mathbb{C}, \forall \varepsilon>0, \mathbb{P}(|X-x|<\varepsilon)>0\} .
$$

Let $Z$ be a solution of (4) having a nonzero expectation. We first prove that $\forall a \in \mathbb{C}, \forall z \in \mathbb{C}$,

$$
[a \in \operatorname{Supp}(A) \text { and } z \in \operatorname{Supp}(Z)] \Longrightarrow a z \in \operatorname{Supp}(Z) .
$$


Indeed, let $\varepsilon>0, a \in \operatorname{Supp}(A)$ and $z \in \operatorname{Supp}(Z)$. Let also $Z_{1}, \ldots, Z_{m}$ be i.i.d. copies of $Z$. Then, with positive probability, $|A-a| \leq \varepsilon$ and $\left|Z_{k}-z\right| \leq \varepsilon$ for any $k$. Therefore, with positive probability,

$$
\left|\sum_{k=1}^{m} V_{k}^{\lambda} Z_{k}-a z\right|=\left|\sum_{k=1}^{m} V_{k}^{\lambda}\left(Z_{k}-z\right)+z(A-a)\right| \leq(m+\varepsilon) \varepsilon+|z| \varepsilon .
$$

Since $\varepsilon$ is arbitrary, this inequality shows that $a z \in \operatorname{Supp}\left(V_{1}^{\lambda} Z_{1}+\cdots+V_{m}^{\lambda} Z_{m}\right)$ which implies that $a z \in \operatorname{Supp}(Z)$ because of $(4)$.

Let $z \in \operatorname{Supp}(Z) \backslash\{0\}$. Such $z$ exist because $\mathbb{E}(Z) \neq 0$. Iterating $(8)$, any complex number of the form $a_{1} \ldots a_{n} z$ where $a_{1}, \ldots, a_{n} \in \operatorname{Supp}(A)$ belongs to $\operatorname{Supp}(Z)$. Therefore, Lemmas 4 and 5 below imply that $\operatorname{Supp}(Z)$ contains $\mathbb{C} \backslash\{0\}$ which suffices to get the conclusion since the support of a probability measure is a closed set.

Lemma 4 There exist $c, c^{\prime} \in \mathbb{C} \backslash\{0\}$ and respective open neighbourhoods $V$ and $V^{\prime}$ of $c$ and $c^{\prime}$ such that $|c|>1,\left|c^{\prime}\right|<1$ and $V \cup V^{\prime} \subseteq \operatorname{Supp}(A)$.

ProOF. Obviously,

$$
\operatorname{Supp}(A)=\left\{\sum_{k=1}^{m} t_{k}^{\lambda}, 0 \leq t_{k} \leq 1, \sum_{k=1}^{m} t_{k}=1\right\}
$$

In particular, $\operatorname{Supp}(A)$ contains the set $f\left([0,1]^{2}\right)$ (the image of $[0,1]^{2}$ by $f$ ), where $f$ is defined by

$$
\begin{aligned}
f:[0,1]^{2} & \rightarrow \mathbb{C} \\
(s, t) & \mapsto(s t)^{\lambda}+(s(1-t))^{\lambda}+(1-s)^{\lambda} .
\end{aligned}
$$

We show that there exist $\left(s_{c}, t_{c}\right)$ and $\left(s_{c^{\prime}}, t_{c^{\prime}}\right)$ in $] 0,1\left[^{2}\right.$ such that $c:=f\left(s_{c}, t_{c}\right)$ and $c^{\prime}:=f\left(s_{c^{\prime}}, t_{c^{\prime}}\right)$ satisfy $|c|>1,0<\left|c^{\prime}\right|<1$ and $f$ is a local diffeomorphism in some respective neighbourhoods of $\left(s_{c}, t_{c}\right)$ and $\left(s_{c^{\prime}}, t_{c^{\prime}}\right)$, which implies the result.

Let $\sigma$ and $\tau$ be respectively the real part and the imaginary part of $\lambda$. By assumption, $0<\sigma<1$. We assume that $\tau>0$; if not, replace $Z$ and $\lambda$ by their conjugates. For any integer $k \geq 1$, denote

$$
u_{k}=\exp \left(-\frac{2 k \pi}{\tau}\right) \text { and } u_{k}^{\prime}=\exp \left(\frac{\pi-2 k \pi}{\tau}\right) .
$$

Then, $u_{k}$ and $u_{k}^{\prime}$ are real numbers in $] 0,1[$ that tend to 0 as $k$ tends to infinity, and they satisfy

$$
\left.u_{k}^{\lambda}=u_{k}^{\sigma} \in\right] 0,1\left[\text { and } u_{k}^{\prime \lambda}=-u_{k}^{\prime \sigma} \in\right]-1,0[
$$

Denote moreover

$$
\left\{\begin{aligned}
s_{k}:=u_{k}+u_{k}^{2}, & t_{k}:=\frac{1}{1+u_{k}} \\
s_{k}^{\prime}:=u_{k}^{\prime}+u_{k}^{\prime 2}, & t_{k}^{\prime}:=\frac{1}{1+u_{k}^{\prime}} .
\end{aligned}\right.
$$

As $0<\sigma<1$, we have

$$
\left|f\left(s_{k}, t_{k}\right)\right|=\left|u_{k}^{\lambda}+u_{k}^{2 \lambda}+\left(1-u_{k}-u_{k}^{2}\right)^{\lambda}\right|=1+u_{k}^{\sigma}+O\left(u_{k}\right)
$$


and

$$
\left|f\left(s_{k}^{\prime}, t_{k}^{\prime}\right)\right|=\left|u_{k}^{\prime \lambda}+u_{k}^{\prime 2 \lambda}+\left(1-u_{k}^{\prime}-u_{k}^{\prime 2}\right)^{\lambda}\right|=1-u_{k}^{\prime \sigma}+O\left(u_{k}^{\prime}\right)
$$

when $k$ tends to infinity, so that $\left|f\left(s_{k}, t_{k}\right)\right|>1$ and $0<\left|f\left(s_{k}^{\prime}, t_{k}^{\prime}\right)\right|<1$ when $k$ is large enough. It remains to show that $f$ is a local diffeomorphism in neighbourhoods of $\left(s_{k}, t_{k}\right)$ and $\left(s_{k}^{\prime}, t_{k}^{\prime}\right)$. Let $\Phi:[0,1]^{2} \rightarrow \mathbb{R}^{2}$ be defined as

$$
\Phi(s, t)=(\Re f(s, t), \Im f(s, t)) .
$$

It suffices to show that the Jacobian of $\Phi$ at suitable $\left(s_{c}, t_{c}\right)$ and $\left(s_{c^{\prime}}, t_{c^{\prime}}\right)$ does not vanish to show that $f$ is a local diffeomorphism at these points. This sufficient condition is equivalent to requiring that

$$
\frac{\partial f}{\partial s} \times \frac{\overline{\partial f}}{\partial t}
$$

is nonreal (the overline denotes the complex conjugacy).

For any $k \geq 1$, after computation, one gets

$$
\begin{array}{r}
\frac{\partial f}{\partial s}\left(s_{k}, t_{k}\right) \times \frac{\overline{\partial f}}{\partial t}\left(s_{k}, t_{k}\right)=|\lambda|^{2} \frac{1+u_{k}}{s_{k} u_{k}}\left[u_{k}^{\lambda}\left(1+u_{k}^{\lambda}\right)-s_{k}\left(1-s_{k}\right)^{\lambda-1}\right]\left[u_{k}^{\bar{\lambda}+1}-u_{k}^{2 \bar{\lambda}}\right] \\
=|\lambda|^{2} \frac{1+u_{k}}{s_{k} u_{k}}\left[u_{k}^{\sigma}+u_{k}^{2 \sigma}-s_{k}\left(1-s_{k}\right)^{\lambda-1}\right]\left[u_{k}^{\sigma+1}-u_{k}^{2 \sigma}\right] .
\end{array}
$$

The above number is real if and only if $\left(1-s_{k}\right)^{\lambda} \in \mathbb{R}$, i.e. if and only if $\tau \log \left(1-s_{k}\right) \in \pi \mathbb{Z}$. Since $s_{k} \neq 0$ and $s_{k}$ tends to zero when $k$ tends to infinity, $\tau \log \left(1-s_{k}\right) \notin \pi \mathbb{Z}$ as soon as $k$ is large enough. Therefore, taking $\left(s_{c}, t_{c}\right)=\left(s_{k}, t_{k}\right)$ for $k$ large enough suffices to get the result. An argument of the same kind applied to the sequence $\left(s_{k}^{\prime}, t_{k}^{\prime}\right)_{k}$ leads to the result on the existence of $\left(s_{c^{\prime}}, t_{c^{\prime}}\right)$.

Lemma 5 Let $V$ and $V^{\prime}$ be respectively open neighbourhoods of $c \in \mathbb{C}$ and $c^{\prime} \in \mathbb{C}$ with $|c|>1$ and $0<\left|c^{\prime}\right|<1$, which do not contain 0 . Let

$$
M:=\left\{v_{1} v_{2} \ldots v_{n}, n \geq 1, v_{1}, v_{2}, \ldots, v_{n} \in V \cup V^{\prime}\right\}
$$

Then $M=\mathbb{C} \backslash\{0\}$.

ProOF. Let $\ell$ and $\ell^{\prime}$ be complex numbers such that $\Re \ell>0$ and $\Re \ell^{\prime}<0$. Let $U$ and $U^{\prime}$ be respectively open neighbourhoods of $\ell$ and $\ell^{\prime}$. Denote by $\mathcal{M}$ the additive submonoid of $\mathbb{C} / 2 i \pi \mathbb{Z}$ generated by $U \cup U^{\prime}$ $\bmod 2 i \pi$; it is the set of classes

$$
\mathcal{M}:=\left\{u_{1}+u_{2}+\cdots+u_{n} \quad \bmod 2 i \pi, n \geq 1, u_{1}, u_{2}, \ldots, u_{n} \in U \cup U^{\prime}\right\} .
$$

We prove hereunder that $\mathcal{M}=\mathbb{C} / 2 i \pi \mathbb{Z}$. Taking the exponential, this suffices to prove the lemma. 


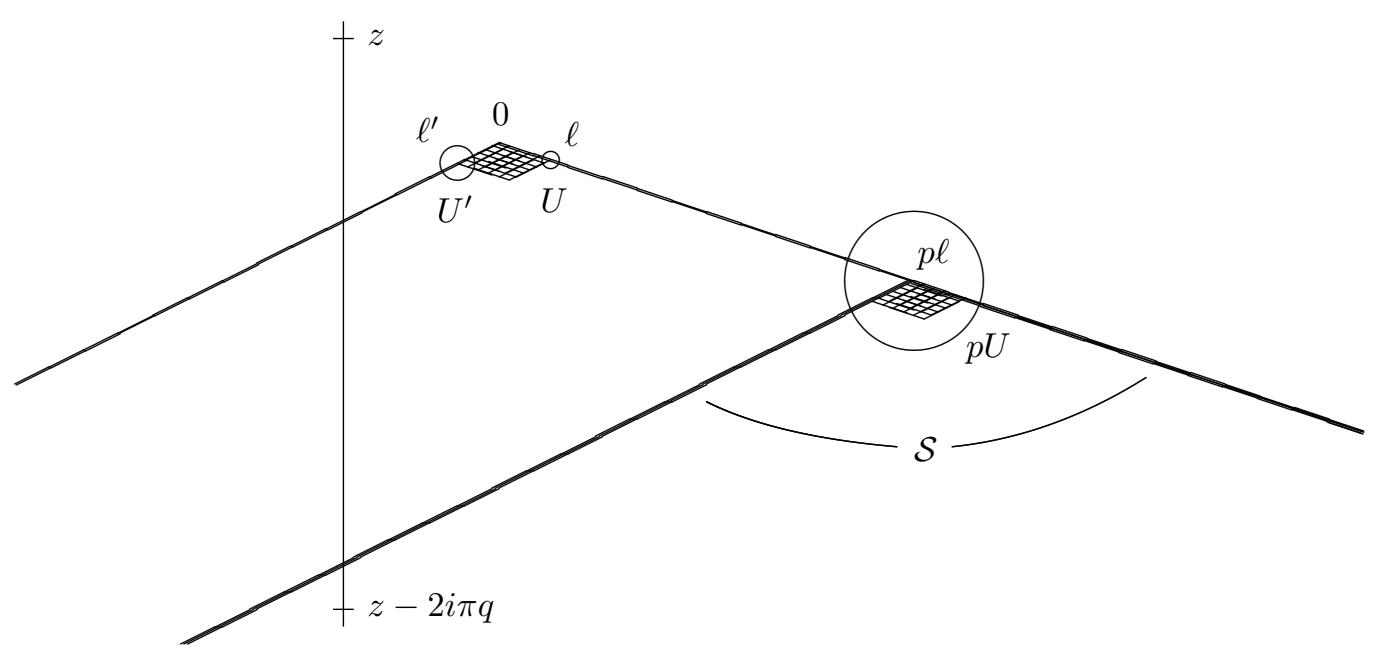

Take an integer $p \geq 1$ large enough so that $p U$ contains a whole mesh of the lattice generated by $\ell$ and $\ell^{\prime}$, i.e. such that $p U \supseteq p \ell+[0,1] \ell+[0,1] \ell^{\prime}$. Then, $\mathcal{M}$ contains the classes $\bmod 2 i \pi$ of the sector $\mathcal{S}:=p \ell+\mathbb{R}_{\geq 0} \ell+\mathbb{R}_{\geq 0} \ell^{\prime}$. Since $\Re \ell \times \Re \ell^{\prime}<0$, when $z$ is any complex number, there exists $q \in \mathbb{Z}$ such that $z-2 i \pi q \in \mathcal{S}$, which proves the result.

\section{Density and exponential moments}

As in the beginning of Section 2 . Theorem[1(ii) and (iii) are straightforward corollaries of the following theorem.

Theorem 6 Let $\lambda$ be a nonreal complex number and $Z$ a solution of (4) having a nonzero expectation.

(i) If $\Re(\lambda)>0$, then $Z$ admits a continuous square integrable density on $\mathbb{C}$.

(ii) If $\Re(\lambda)>\frac{1}{2}$, then $\mathbb{E} e^{\delta|Z|}<\infty$ for some $\delta>0$. The exponential moment generating series of $Z$ (thus) has a positive radius of convergence.

PROOF. It runs along the same lines as in [CLP11] and uses the Fourier transform $\varphi$ of $Z$, namely

$$
\varphi(t):=\mathbb{E} \exp \{i\langle t, Z\rangle\}=\mathbb{E} \exp \{i \Re(\bar{t} Z)\}, \quad t \in \mathbb{C},
$$

where $\langle x, y\rangle=\Re(\bar{x} y)=\Re(x) \Re(y)+\Im(x) \Im(y)$. In terms of Fourier transforms, Eq. (4) reads

$$
\varphi(t)=\mathbb{E}\left(\prod_{k=1}^{m} \varphi\left(t V_{k}^{\bar{\lambda}}\right)\right) .
$$

- To get (i), we prove that $\varphi$ is in $L^{2}(\mathbb{C})$ because it is dominated by $|t|^{-\delta}$ for some $\delta>1$ so that the inverse Fourier-Plancherel transform provides a square integrable density for $Z$. The guiding idea consists in adapting methods (developed in [Liu99] and [Liu01]) usually applied to positive real-valued 
random variables to the present complex-valued case. For any $r \geq 0$, denote

$$
\psi(r):=\max _{|t|=r}|\varphi(t)| .
$$

Using Theorem 3, one can step by step mimick the proof of Theorem 7.17 in [CLP11] to get the result. We just give hereunder an overview of this proof, written as a succession of hints.

Show first that Theorem 3 implies that $\psi(r)<1$ for any $r>0$. Then, notice that

$$
\psi(r) \leq \mathbb{E}\left(\prod_{k=1}^{m} \psi\left(r\left|V_{k}\right|^{\sigma}\right)\right) .
$$

By Fatou's lemma, 10$]$ implies that $\lim _{\sup _{+\infty}} \psi(r) \in\{0,1\}$. Iterating suitably inequality 10$]$ leads to $\lim _{+\infty} \psi(r)=0$. Finally, applying 10 again we can show that $\psi(r)=O\left(r^{-\delta}\right)$ for some $\delta>1$, so that $\varphi$ is square integrable on $\mathbb{C}$, which leads to the result.

- To get (ii), like in [CLP11], we use Mandelbrot's cascades. Denote $\mathcal{V}:=\left(V_{1}, V_{2}, \ldots, V_{m}\right)$. Let $U$ be the set of finite sequences of positive integers between 1 and $m$, namely

$$
U:=\bigcup_{n \geq 1}\{1,2, \ldots, m\}^{n} .
$$

Elements of $U$ are denoted by concatenation. Let $\mathcal{V}_{u}:=\left(V_{u 1}, V_{u 2}, \ldots, V_{u m}\right), u \in U$ be independent copies of $\mathcal{V}$, indexed by all finite sequences of integers $u=u_{1} \ldots u_{n} \in U$.

Introduce the martingale $\left(Y_{n}\right)_{n \geq 1}$ defined by

$$
Y_{n}:=\sum_{u_{1} \ldots u_{n} \in\{1, \ldots, m\}^{n}} V_{u_{1}}^{\lambda} V_{u_{1} u_{2}}^{\lambda} \ldots V_{u_{1} \ldots u_{n}}^{\lambda} .
$$

By $(7), \mathbb{E}\left(Y_{n}\right)=\mathbb{E}(A)=1$. It can be easily seen that

$$
Y_{n+1}=\sum_{k=1}^{m} V_{k}^{\lambda} Y_{n, k}
$$

where $Y_{n, k}$ for $1 \leq k \leq m$ are independent of each other and independent of the $V_{k}$ and each has the same distribution as $Y_{n}$. Besides, since $\sigma>\frac{1}{2}, m \mathbb{E} V_{1}^{2 \sigma}<1$ and, by Cauchy-Schwarz inequality,

$$
\mathbb{E}|A|^{2} \leq \mathbb{E}\left(\sum_{k=1}^{m}\left|V_{k}^{\lambda}\right|\right)^{2}=\mathbb{E}\left(\sum_{k=1}^{m} V_{k}^{\sigma}\right)^{2} \leq 2 \mathbb{E} \sum_{k=1}^{m} V_{k}^{2 \sigma}=2 m \mathbb{E} V_{1}^{2 \sigma}<2 .
$$

Therefore for $n \geq 1, Y_{n}$ is square integrable and

$$
\operatorname{Var} Y_{n+1}=\left(\mathbb{E}|A|^{2}-1\right)+m \mathbb{E} V_{1}^{2 \sigma} \operatorname{Var} Y_{n},
$$

where $\operatorname{Var} X=\mathbb{E}\left(|X-\mathbb{E} X|^{2}\right)$ denotes the variance of $X$. Thus, the martingale $\left(Y_{n}\right)_{n}$ is bounded in $L^{2}$, so that when $n \rightarrow+\infty$,

$$
Y_{n} \rightarrow Y_{\infty} \text { a.s. and in } L^{2},
$$


where $Y_{\infty}$ is a (complex-valued) random variable with variance

$$
\operatorname{Var}\left(Y_{\infty}\right)=\frac{\mathbb{E}|A|^{2}-1}{1-m \mathbb{E} V_{1}^{2 \sigma}}
$$

Passing to the limit in Eq. (11) shows that $Y_{\infty}$ is a solution of Eq. (4) and by unicity, (ii) in Theorem 6 holds as soon as it holds for $Y_{\infty}$.

This last fact comes from an adaptation of Lemma 8.29 in [CLP11], giving some constants $C>0$ and $\varepsilon>0$ such that for all $t \in \mathbb{C}$ with $|t| \leq \varepsilon$, we have

$$
\mathbb{E} e^{\left\langle t, Y_{\infty}\right\rangle} \leq e^{\Re(t)+C|t|^{2}} .
$$

The adaptation relies on $\sum_{k=1}^{m} V_{k}^{2 \sigma}<1$ a.s. for $\sigma>\frac{1}{2}$. The last assertion implies that $\mathbb{E} e^{t\left|Y_{\infty}\right|<\infty}$ for $t>0$ small enough, so that the exponential moment generating series of $Y_{\infty}$ has a positive radius of convergence.

\section{References}

[CH01] H.-H. Chern and H.-K. Hwang. Phase changes in random $m$-ary search trees and generalized quicksort. Random Structures and Algorithms, 19:316-358, 2001.

[CLP11] B. Chauvin, Q. Liu, and N. Pouyanne. Limit distributions for multitype branching processes of m-ary search trees. arXiv:1112.0256v1, math.PR, 2011.

[CP04] B. Chauvin and N. Pouyanne. m-ary search trees when $\mathrm{m}>26$ : a strong asymptotics for the space requirements. Random Structures and Algorithms, 24(2):133-154, 2004.

[FK04] J.A. Fill and N. Kapur. The space requirement of $m$-ary search trees: distributional asymptotics for $m \geq 27$. Proceedings of the 7th Iranian Conference, page arXiv:math.PR/0405144, 2004.

[Hen91] P. Hennequin. Analyse en moyenne d'algorithme, tri rapide et arbres de recherche. PhD Thesis, Ecole Polytechnique, 1991.

[Hwa03] H.-K. Hwang. Second phase changes in random m-ary search trees and generalized quicksort: convergence rates. Ann. Probab., 31, 2:609-629, 2003.

[Jan04] S. Janson. Functional limit theorem for multitype branching processes and generalized Pólya urns. Stochastic Processes and their Applications, 110:177-245, 2004.

[Liu99] Q. Liu. Asymptotic properties of supercritical age-dependent branching processes and homogeneous branching random walks. Stochastic Processes and their Applications, 82(1):61-87, 1999.

[Liu01] Q. Liu. Asymptotic properties and absolute continuity of laws stable by random weighted mean. Stochastic Processes and their Applications, 95:83-107, 2001.

[Mah92] H.M. Mahmoud. Evolution of Random Search Trees. John Wiley \& Sons Inc., New York, 1992.

[Pou05] N. Pouyanne. Classification of large Pólya- Eggenberger urns with regard to their asymptotics. Discrete Mathematics and Theoretical Computer Science, AD, pages 275-286, 2005. 
